\title{
Capital culturel et identité sociale : les fonctions sociales du discours sur l'encombrement des professions libérales au XiXe siècle
}

\section{Cultural Capital and Social Identity: Social Functions of the Discourse on the Saturation in the Liberal Professions in the 19th Century}

\section{Robert GAGNON}

Volume 21, numéro 2, automne 1989

La culture comme capital

URI : https://id.erudit.org/iderudit/001268ar

DOI : https://doi.org/10.7202/001268ar

Aller au sommaire du numéro

\section{Éditeur(s)}

Les Presses de l'Université de Montréal

ISSN

0038-030X (imprimé)

1492-1375 (numérique)

Découvrir la revue

Citer cet article

GAGNON, R. (1989). Capital culturel et identité sociale : les fonctions sociales du discours sur l'encombrement des professions libérales au XiXe siècle. Sociologie et sociétés, 21(2), 129-146. https://doi.org/10.7202/001268ar

\section{Résumé de l'article}

Cet article a pour objet le discours sur l'encombrement des professions libérales au XIXe siècle. Au lieu de s'interroger sur le nombre des avocats, médecins ou notaires, afin de déterminer si ces professions connaissent un "encombrement" au cours du XIXe siècle, l'auteur suggère plutôt que l'encombrement des professions libérales dont font état plusieurs membres de ces professions est en fait une construction sociale. Dans la première moitié du XIXe siècle, ce discours est relié au travail de désignation entrepris par les membres des professions libérales afin de se doter d'une identité sociale commune: celle de l'honnête homme. Plus tard, dans la deuxième moitié du XIXe siècle, ce discours remplit une tout autre fonction sociale. L'auteur rapporte le discours sur l'encombrement des professions libérales aux stratégies de reproduction de groupes désormais formés. La multiplication des collèges et le monopole de l'éducation classique sur le système d'enseignement secondaire au Québec, en prédisposant les étudiants à la prêtrise ou aux professions libérales, menacent la valeur sociale et économique de ces titres professionnels. L'encombrement des professions libérales est alors invoqué pour restreindre l'accès aux collèges classiques, notamment en promouvant la mise en place d'une nouvelle filière dans l'enseignement secondaire: celle de l'éducation pratique.
Tous droits réservés @ Les Presses de l'Université de Montréal, 1989
Ce document est protégé par la loi sur le droit d'auteur. L'utilisation des services d’Érudit (y compris la reproduction) est assujettie à sa politique d'utilisation que vous pouvez consulter en ligne.

https://apropos.erudit.org/fr/usagers/politique-dutilisation/ 


\section{Capital culturel et identité sociale: les fonctions sociales du discours sur l'encombrement des professions libérales au XIX ${ }^{\mathrm{e}}$ siècle}

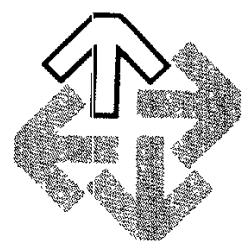

ROBERT GAGNON ${ }^{1}$

Au Québec, les membres des professions libérales commencent, dès le début du $\mathrm{XIX}^{\mathrm{e}}$ siècle, à jouer un rôle déterminant, tant au plan social que politique. Plusieurs historiens ont cherché à évaluer leur nombre; ils ont également essayé de comprendre les conditions de leur participation active à la vie politique et sociale au Canada français ${ }^{2}$. D'autres historiens et certains sociologues se sont quant à eux interrogés sur la position de classe de ceux qu'ils ont identifiés comme la «petite bourgeoisie professionnelle» ${ }^{3}$. Finalement, des études, s'inscrivant dans le champ de la sociologie des professions, ont porté sur le processus de professionnalisation que connaissent certaines occupations au $\mathrm{XIX}^{e}$ siècle, notamment la médecine ${ }^{4}$. Toutefois, les problématiques privilégiées par ces auteurs les ont amenés à négliger les rapports entre l'ascension sociale des professions libérales et la mise en place d'un système d'enseignement secondaire essentiellement constitué par les collèges classiques qui s'érigent un peu partout au Québec. Les travaux de Pierre Bourdieu sur les stratégies de reproduction sociale ${ }^{5}$ et ceux de Luc Boltanski sur la formation d'un groupe social comme celui des cadres ${ }^{6}$, nous permettent cependant de mieux comprendre la contribution que le système d'enseignement apporte à la production et à la reproduction des rapports de force entre les différents groupes sociaux.

Four sortir des débats sans fin et sans solution sur la «position de classe» des cadres, Boltanski renonce à donner une «définition préalable» de ce groupe. Il prend plutôt comme

1. L'auteur tient à remercier Yves Gingras pour ses précieux commentaires.

2. On pense surtout à F. OUELLET (1971), Histoire économique et sociale du Québec, 1760-1850, Montréal, Fides, 2 vols.

3. G. BOURQUE (1970), Classes sociales et question nationale, 1760-1840, Montréal, Parti Pris. P.-A. LINTEAU, «Quelques réflexions autour de la bourgeoisie québécoise, 1850-1914», in Revue d'Histoire de l'Amérique française, vol. XXX, $\mathrm{n}^{\circ} 1$, (juin 1976), pp. 55-56.

4. J. BERNIER (1989), La médecine au Québec, Québec, Presses de 1'Université Laval.

5. P. BOURDIEU, «Reproduction culturelle et reproduction sociale», in Informations sur les sciences sociales, vol. $X, \mathrm{n}^{\circ} 1,1971$, pp. 45-79. P. BOURDIEU, L. BOLTANSKI, M. de SAINT MARTIN, «Les stratégies de reconversion», in Informations sur les sciences sociales, vol. XII, n ${ }^{\circ} 5,1973$, pp. 61-113.

6. L. BOLTANSKI (1982), Les cadres: la formation d'un groupe social, Paris, Minuit. 
objet la conjoncture historique dans laquelle les cadres se sont regroupés, et se sont dotés d'un nom, d'organisations, de porte-parole, de systèmes de représentations et de valeurs. Il tente alors de rendre compte de l'identité sociale de ce groupe en analysant le travail

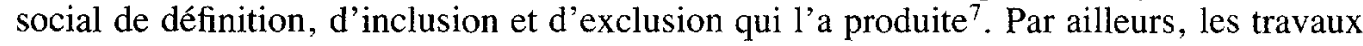
de Bourdieu et de ses collaborateurs fournissent une série de concepts, fort éclairants, sur les mécanismes les plus subtils par lesquels la structure des rapports de forces et des rapports symboliques entre les classes persiste et se reproduit de génération en génération ${ }^{8}$. Pour éclairer ces mécanismes de domination, Bourdieu prend pour objet les conditions sociales de production et de distribution des ressources culturelles. Il montre ainsi comment les institutions culturelles et la production des définitions légitimes du monde social confinent les individus et les groupes dans des structures hiérarchiques de domination et de subordination. À la lumière de ces travaux, se révèle le rôle déterminant du système d'enseignement. En effet, le système d'enseignement, en tant qu'instance de production des garanties scolaires, c'est-à-dire de titres scolaires pouvant permettre l'accession à des positions dominantes dans la société, devient dans les pays qui s'industrialisent un enjeu important de lutte entre les classes ${ }^{9}$.

Il nous est permis de supposer qu'au XIX ${ }^{\mathrm{e}}$ siècle, la mise en place d'un système d'enseignement secondaire et supérieur, au Québec, permet à certains groupes de se doter d'une identité, fondée en grande partie sur l'acquisition de dispositions essentiellement culturelles. Un aspect important de l'identité sociale d'un groupe, comme celui des médecins, avocats ou notaires, se construit en effet dans la première moitié du XIX ${ }^{\mathrm{e}}$ siècle. Dans cet article, nous nous attarderons plus spécifiquement sur un moment précis de ce processus de construction d'une identité sociale. En prenant pour objet le discours sur l'encombrement des professions libérales qui s'instaure au début du XIX ${ }^{\mathrm{e}}$ siècle et perdure par la suite, nous analyserons les fonctions sociales de ce discours en le rapportant au travail social de définition qui a accompagné la formation de ces groupes. Nous verrons ainsi comment, en véhiculant ce discours, certains médecins, avocats ou notaires vont permettre aux membres de ces professions de se doter d'une identité commune, principalement en utilisant les collèges classiques. Dans la seconde moitié du XIX ${ }^{\mathrm{e}}$ siècle, le discours sur l'encombrement des professions libérales remplira cependant une nouvelle fonction sociale. En effet, ce discours servira, entre autres, à exiger une réforme du système d'enseignement franco-catholique, afin de restreindre l'accès aux professions libérales, en diversifiant l'enseignement secondaire dont l'unique filière était le collège classique.

On comprendra qu'il ne s'agit pas, ici, de s'interroger sur l'existence réelle ou non de «l'encombrement» des professions libérales, car l'importance de ce discours, selon nous, réside moins dans sa valeur de vérité, que dans sa capacité à défendre les intérêts des groupes qui le propagent. Ce discours est tout d'abord présent dans les journaux de l'époque. Des députés à l'Assemblée nationale font également état de «l'encombrement» des professions libérales. À la fin du siècle, lorsque sont créées les premières revues professionnelles, des porte-parole des professions libérales se prononceront alors sur ce sujet. Toutefois ces sources ne nous révèlent pas nécessairement les enjeux réels de ce discours. C'est tout d'abord à l'Assemblée législative où sont présentés des projets de loi concernant l'incorporation des professions libérales et, ensuite, dans les rapports des ministres de l'Instruction publique que l'on peut percevoir les fonctions que remplit ce discours. Par ailleurs, sans qu'il soit nécessaire de parler de deux solitudes, il faut admettre tout de même qu'un certain clivage existe entre les membres des professions libérales,

7. L. BOLTANSKI, op. cit., p. 51.

8. P. BOURDIEU, J.-C. PASSERON (1970), La reproduction: éléments d'une théorie du système d'enseignement, Paris, Minuit.

9. P. BOURDIEU, L. BOLTANSKI, «Le titre et le poste» in Actes de la recherche en sciences sociales, $\mathrm{n}^{\circ} 2,1975, \mathrm{pp} .95-107$. 
clivage fondé sur leur appartenance à l'un ou l'autre des deux principaux groupes ethniques du Québec. Ainsi, les fonctions sociales du discours sur l'encombrement des professions libérales sont étroitement liées au système d'éducation franco-catholique. C'est pourquoi nous restreignons ici notre analyse aux médecins, notaires et avocats canadiens-français.

\section{LA VALEUR DE L'ÉDUCATION DES PROFESSIONS LIBÉRALES}

C'est à la faveur d'une conjoncture historique particulière que les conditions de l'ascension sociale et politique des professions libérales sont réunies. $\grave{A}$ la fin du XVIII ${ }^{\mathrm{e}}$ siècle, alors que la constitution de 1791 favorise l'émergence des partis politiques et le recrutement de leurs membres et de leurs leaders dans les couches populaires de la sociéte ${ }^{10}$, bon nombre de membres des professions libérales envahissent la Chambre d'Assemblée. Dès le début du XIX ${ }^{\mathrm{e}}$ siècle, les seigneurs sont, à toutes fins pratiques, margiaalisés à la Chambre d'Assemblée. Leur influence ne se fera désormais sentir qu'au conseil législatif. La domination des notaires, médecins et avocats à la Chambre d'Assemblée (lieu stratégique par excellence puisqu'il permet de parler au nom de la nation et ainsi de convertir les intérêts d'un groupe en intérêts nationaux) se fait alors sentir dès la première décennie du XIX ${ }^{\mathrm{e}}$ siècle $^{11}$.

N'oublions pas non plus que l'ascension politique des avocats, médecins et notaires va de pair avec une croissance rapide de leurs effectifs, qui se traduit par l'émergence d'une conscience de classe dont on peut retracer l'évolution dans les différents discours que prononcent leurs porte-parole. Cette conscience de classe est alors identifiée à une conscience nationale. Les membres des professions libérales se perçoivent comme les élites de la nation canadienne-française et les défenseurs privilégiés des intérêts des Canadiens français contre les marchands et bureaucrates anglais, bref contre la puissante minorité anglaise ${ }^{12}$.

Au même moment, un système d'enseignement classique mis en place et contrôlé par le clergé, s'érige pour constituer l'unique filière de l'enseignement secondaire. La croissance démographique et la pénurie de prêtres favorisent, dès le début du XIX ${ }^{\mathrm{e}}$ siècle, la création des collèges classiques. Aux petits séminaires de Québec (1668) et de Montréal (1767) viennent s'ajouter ceux de Nicolet (1803), St-Hyacinthe (1811), et Ste-Thérèse (1825), tout comme les collèges de St-Denis-sur-Richelieu (1805), St-Roch (1818), Chambly (1825) et Ste-Anne de La Pocatière (1827). Le type même de formation, à caractère humariste, que dispense le collège, profite aux membres des professions libérales qui, tout au long du XIX ${ }^{\mathrm{e}}$ siècle, intensifient leurs efforts afin d'exiger, comme préalable à la profession, les études classiques. En effet, instrument de reproduction sociale du clergé, le collège classique, dans la première moitié du $\mathrm{XIX}^{\mathrm{e}}$ siècle, contribue peu à peu à l'éducation d'un nouveau groupe, il s'agit bien sûr des membres des professions libérales qui, grâce à cette instance de production et de reproduction sociale, apparaîtront de plus en plus comme une classe de gens distingués et distinguables. On sait que le collège recrute: ses étudiants principalement chez les fils d'agriculteurs dont plus de la moitié trouvent leur ascension sociale dans la prêtrise ${ }^{13}$, tandis que certains se dirigent vers les seules carrières auxquelles cette institution les destine: les professions libérales.

Du côté des professions libérales, il est clair que l'une des stratégies par lesquelles leurs membres réussissent à s'unir par des propriétés d'habitus, est certainement celle qui consiste à utiliser le collège comme un instrument privilégié de socialisation. En se donnant

208.

10. F. OUELLET (1972), Éléments d'histoire sociale du Bas-Canada, Montréal, Hurbutise HMH, p.

11. Voir à ce sujet le chapitre «La crise sous Craig (1807-1811): nature des conflits et historiographie» dans le livre de J. P. WALLOT, Un Québec qui bougeait, Montréal, Boréal Express, 1973, pp. 143-167.

12. F. OUELLET, op. cit., vol. II, pp. 581-582.

13. C. GALARNEAU (1978), Les collèges classiques au Canada français, Montréal, Fides, p. 146. 
les moyens de produire une perception commune des professions libérales, ses membres se dotent de propriétés secondaires indispensables pour s'assurer en toute légitimité une position dominante dans l'espace social. Ils pourront désormais apparaitre sur la scène sociale comme un groupe homogène, c'est-à-dire une classe de gens, non seulement définis par leur occupation, mais surtout par des caractéristiques auxiliaires qui sont, en fait, celles de «l'honnête homme». B. Tunis a ainsi remarqué, très justement, qu'après 1795 , de plus en plus de médecins canadiens-français passent par le collège classique avant leur apprentissage ou leurs études médicales. Selon elle, cette nouvelle trajectoire de la formation des médecins francophones leur assure une meilleure représentation dans le milieu urbain par rapport aux médecins anglophones, ce qui renforce leur influence au niveau politique ${ }^{14}$. En parlant de la période 1810-1820, Tunis affirme: «expansion in numbers and improved education was also accompanied by increased political and social awareness steming from their close relationship of the medical profession to the other liberal professions ${ }^{15}$.

La quête collective du statut privilégié de ces professions passe donc inséparablement par l'acquisition d'une éducation qui soit à la hauteur des aspirations de ces groupes sociaux. Au début du XIX ${ }^{e}$ siècle, toutefois, c'est encore l'ordonnance de 1785 qui réglemente les professions d'avocat et de notaire. Cette première loi, organisant le notariat et la profession d'avocat sous le régime anglais, oblige les aspirants à ces professions à faire une cléricature de cinq années chez un patron avocat ou notaire. À la fin de sa cléricature, le candidat subit un examen devant «quelques-uns des anciens notaires ou praticiens». Ensuite, le «patron délivrera un certificat de capacité et de bonne mours» ${ }^{16}$. En ce qui concerne les médecins, c'est un acte de loi de 1788 qui régit cette profession. La licence de médecin est décernée par un bureau d'examinateurs aux aspirants médecins ou chirurgiens. Certains praticiens ont été formés dans une école de médecine aux ÉtatsUnis ou en Europe, tandis que d'autres sont encore formés par l'apprentissage chez un patron médecin ${ }^{17}$.

Comme partout ailleurs, aux États-Unis comme en Europe, aucun préalable scolaire n'est donc encore exigé pour ceux qui désirent embrasser les carrières de médecin, d'avocat ou de notaire. En fait, aucune éducation garantissant les qualités «morales», «intellectuelles» ou «scientifiques» n'est alors exigée des membres des professions libérales. Comme le début du XIX ${ }^{\mathrm{e}}$ siècle est témoin de l'ascension de ce type de professions, dont on peut supposer que la majorité des membres sont issus des classes populaires, il n'est nullement surprenant que la valeur de l'éducation de ces groupes en formation se révèle, dès cette époque, comme un enjeu majeur dans la lutte entre différents groupes pour conserver ou améliorer leur position dans l'espace social, notamment entre les membres de la bureaucratie anglaise, les seigneurs et les membres des professions libérales.

On comprend dès lors l'attitude du gouverneur Craig, en 1808, devant l'arrivée de plusieurs avocats et notaires à la Chambre d'Assemblée qui écrit: «Les Canadiens sont insubordonnés et n'aiment pas la soumission. Si les seigneurs possédaient leur ancienne influence, il en serait autrement ${ }^{18}$. De plus, ces avocats et notaires ont conscience de leurs intérêts, ce qui les rend d'autant plus menaçants. Craig le perçoit bien lorsqu'il ajoute: «Les membres élus à la dernière Chambre d'Assemblée comprennent quinze hommes de loi, quatorze cultivateurs et seulement sept seigneurs. Les avocats forment

14. B. TUNIS (1979), The Medical Profession in Lower Canada: Its Evolution as a Social Group, 1788-1838, mémoire de maîtrise, Université Carleton, p. 52.

15. Ibid, p. 61

16. J. E. ROY (1900), Histoire du notariat au Canada, 4 vol., Lévis, vol. II, p. 229.

17. Pour plus de détails sur l'apprentissage et les exigences requises à l'entrée dans cette profession voir J. BERNIER, «François Blanchet et le mouvement réformiste en médecine au début du XIX ${ }^{\mathrm{e}}$ siècle» in $R H A F$, vol. XXXIV, no 2, 1980, pp. 223-244.

18. Citée par J. E. ROY, op. cit., vol. II, p. 229. 
un parti puissant dans la Chambre; ils sont toujours ensemble, et ils pensent, en opposant les vues du gouvernement, de le forcer à leur donner des places» ${ }^{19}$. Craig invoque la valeur douteuse de l'éducation de ces derniers afin de contrer les efforts d'un groupe qui, par la conscience même de ses intérêts, se définit de plus en plus comme une classe particulière. En 1810, il écrit à Lord Liverpool: «La Chambre, pour la partie canadienne, est remplie plus que jamais par des avocats, des notaires, des marchands et des habitants, c'est-ì-dire la classe la plus ignorante des cultivateurs [...] Cette classe d'avocats et de notaires sans principes ignore complètement la constitution anglaise $[\ldots]{ }^{20}$.

Plusieurs membres des professions libérales ne restent pas insensibles à la perception qu'ont d'eux certains membres influents de la société. Il devient urgent de s'assurer que n'importe quel individu, sachant tout juste «griffonner», ne puisse pas accéder à de si «nobles» professions. Ce n'est pas par hasard si l'année 1808 voit un notaire influent de la Chambre d'Assemblée, Jean-Marie Mondelet, présenter un projet de loi ayant pour objectif d'inciter les aspirants au notariat et à la profession d'avocat à passer par le collège classique.

Les principes, dit-il, en proposant cette mesure, sont d'établir plus particulièrement les qualifications des aspirants; de n'ouvrir la porte de la profession de notaire qu'à ceux dont les mœurs, la capacité et l'intégrité répondront à l'importance des devoirs qu'ils auront à remplir [...]. Mon but est de faire revivre les idées avantageuses que l'on avait autrefois de cette noble profession, en y admettant, par la suite, que des personnes dont l'habileté et la régularité des mœurs forment le caractère distingué ${ }^{21}$.

La proposition de Mondelét incita M. de Salaberry, arpenteur, à proposer, lui aussi, un projet de loi pour réglementer sa profession. La présentation de ces projets de loi suscite à l'époque une certaine réaction. On peut lire alors, dans un journal de l'époque, les premiers témoignages faisant état de l'ignorance des notaires, avocats et arpenteurs qui sont autant d'appuis en faveur de l'adoption de ces projets de lois ${ }^{22}$. Ces projets de loi ne seront pas adoptés (celui de Mondelet, bien qu'accepté par la Chambre d'Assemblée, fut soumis à la consultation par le Conseil législatif et ne fut jamais adopté), il n'en demeure pas moins qu'ils sont des indicateurs de la volonté naissante, au sein de certains membres des professions libérales, de se forger une identité à l'image de l'honnête homme. Du côté des médecins et chirurgiens, la volonté de rehausser le niveau d'éducation des aspirants à la profession se manifeste également. Encore là, c'est en dénonçant l'ignorance de certains d'entre eux, que des médecins réclament une éducation à la hauteur d'une autre «noble» profession. Il n'est pas rare de lire, dans les journaux de la première moitié $\mathrm{du} \mathrm{XIX}^{\mathrm{e}}$ siècle, des propos comme ceux de ce médecin outré par le peu de rigueur dont font preuve les examinateurs dans l'octroi des licences:

the health and lives of the inhabitants of many of the most populous and wealthy sections of the province, [are] generally at the mercy of a sordid class of men, totally devoid of education $[\ldots]^{23}$

Pour comprendre l'importance des débats qui s'instaurent, au début du XIX ${ }^{e}$ siècle, sur la valeur de l'éducation des professions libérales, il faut les rapporter au travail social de définition, entrepris par les porte-parole de ces groupes. Les cas de notaires illettrés,

19. Ibid, p. 229-230.

20. Ibid, p. 230. Voir aussi J. P. WALLOT, «La crise sous Craig...» op. cit., p. 151.

21. Mondelet complétera ses premières remarques, après qu'un comité aura été formé pour étudier son projet, en affirmant entre autres: «N'est-il pas injuste de mettre sur le même pied un jeune homme qui a étudié les belles-lettres pendant plusieurs années, et un jeune homme qui n'a jamais appris qu'à lire et à écrire?\% Courrier de Québec, 13 février 1808, p. 14.

22. Courrier de Québec, 13 février 1808, p. 19, 20 février 1808, p. 27.

23. Cité par R. HAMOWY (1984), Canadian Medicine: A study in Restricted Entry, Fraser Institute, p. 30 . 
d'avocats incultes, ou de médecins sans mœurs, sont relevés par certains membres de ces professions pour confirmer, en fait, les propriétés contraires attachées aux professions libérales, à savoir bonnes mœurs, érudition et intégrité. Il ne s'agit pas, ici, de vérifier la véracité de ce discours ou de jauger l'ignorance et le niveau de la culture des membres des professions libérales, au début du XIX ${ }^{\mathrm{e}}$ siècle, mais de montrer plutôt que ce discours $s^{\prime}$ inscrit dans une stratégie collective de ces groupes pour se donner des attributs distinctifs qui leur permettront d'améliorer leur position dans la hiérarchie des groupes sociaux. En somme, la force et la récurrence du discours sur l'encombrement des professions libérales qui va apparaître, au cours des années 1820 , tient au fait qu'il a pour enjeu principal les mécanismes de production et de reproduction sociales de ces groupes.

\section{LA CONSTRUCTION D'UNE IDENTITÉ: «L'HONNÊTE HOMME»}

On sait maintenant que l'une des fonctions essentielles du collège classique est de produire des rapports sociaux ${ }^{24}$. En effet, le collège est avant tout une structure d'incorporation de pratiques spécifiques qui génèrent un style de vie particulier. Le collège est donc beaucoup plus qu'une institution où l'on transmet des connaissances; c'est un lieu où l'on assimile un système de schèmes générateurs de pratiques et un système de classement des pratiques qui font voir les dépositaires de ces dispositions particulières comme des êtres partageant un même style de vie, ayant une même vision du monde ${ }^{25}$.

Ainsi, le collège initie l'étudiant à entretenir un rapport au corps spécifique. On lui apprend la façon de soigner son corps par un régime de vie convenable et de le développer par des exercices physiques. C'est encore au collège que l'étudiant acquiert une manière d'être distinctive, qui passe par la connaissance et la reconnaissance des règles de bienséance, d'hygiène et de civilité, nécessaires à un style de vie qui apparaît aussi comme un art de vivre. Le collège est aussi un endroit où la distance entre le parler populaire et le parler légitime est entretenue. La correction du langage par des exercices de diction ou de phonétique fait partie de l'enseignement des collèges, tout comme l'art oratoire et le théâtre. Si la présentation de soi, grâce à un rapport au corps particulier, confère à l'étudiant les allures extérieures de l'honnête homme, il reste à lui procurer toutes les propriétés pertinentes nécessaires au raffinement de cette image. On retrouve dans ces institutions les conditions essentielles pour façonner une sensibilité aux cuvres d'art légitimes, en développant les modes d'expression de la beauté: les beaux-arts, la musique et les arts plastiques. Aux côtés des matières académiques que sont le grec et le latin, la grammaire française et l'anglais, la théologie et la philosophie, l'histoire et la géographie, les mathématiques et parfois les sciences, s'enseigne donc un art de vivre légitime, distinct et opposé, à bien des égards, à la manière d'être populaire. Comme le dit si bien, en 1827, un lecteur de La Minerve, le collège classique est «un sanctuaire où [l'étudiant] est initié aux mystères des jouissances idéales, et où on lui montre de loin la gloire et l'honneur dont il peut se couvrir. C'est de nos collèges que sont sortis presque tous les citoyens marquants qui ont servi leur pays, soit dans la législature ou ailleurs» ${ }^{26}$.

Comme, en ce début de XIX ${ }^{\mathrm{e}}$ siècle, le chemin qui mène aux carrières de médecin, d'avocat ou de notaire ne passe pas nécessairement par le collège classique, ni aucune autre institution scolaire, plusieurs membres des professions libérales commencent bientôt à faire remarquer que n'importe quel citoyen sachant lire et écrire, et ne possédant aucune sorte d'éducation, peut accéder aux «nobles» professions libérales. Dès la fin des années

24. C. GALARNEAU, op. cit., pp. 201-217.

25. En fait ce qui prend forme chez les individus formés par les collèges, c'est ce que Pierre Bourdieu nomme l'habitus. Ce concept sociologique fait partie d'une théorie de la pratique esquissée par Bourdieu dans Le sens pratique, Paris, Minuit, 1980. L'application de ce concept dans la construction de la classe sociale se retrouve dans l'ouvrage du même auteur La distinction, Paris, Minuit, 1979.

26. La Minerve, 5 juillet 1827, p. 3 . 
1820, plusieurs lecteurs de La Minerve commencent à acheminer des lettres ouvertes qui font état de la situation «déplorable» dans laquelle se retrouvent alors les professions libérales au Bas-Canada.

Ces premiers commentaires, qui soulèvent pour la première fois la question de l'encombrement des professions libérales, prennent alors l'allure d'un débat qui, parmi les membres de ces professions, s'instaure autour de la valeur de leur titre sur le marché du travail, de l'organisation de leur profession et, finalement, de la valeur de l'éducation de leurs membres, débat dont l'enjeu ultime est la définition même de la profession et, plus encore, de ceux qui la pratiquent.

La première lettre, dans La Minerve ${ }^{27}$, qui fait mention de l'encombrement des professions libérales, se veut une analyse de la nouvelle situation dans laquelle se trouvent les professions libérales. L'auteur, sous le pseudonyme de Sincerus, mentionne que si «les professions d'avocat, notaire et médecin étaient, il y a cinquante ans, fort lucratives et très profitables» la situation est tout autre maintenant. Ces professions «sont à présent les moins avantageuses de notre pays». La raison en est que la rareté que connaissaient ces professions antérieurement n'existe plus. Il y a un trop grand nombre d'avocats et de médecins. Pour le signataire de cette lettre, «il est douloureux qu'une profession qui est en ellè-même si honorable, soit tombée par les circonstances, dans une espèce de dégradation qui la rend moins respectable et moins avantageuse». Ainsi le nombre grandissant de notaires, selon lui, n'entraîne pas seulement une dévaluation économique de la valeur du titre professionnel, mais aussi, et surtout une dévaluation sociale. La thèse de ce correspondant (sur la situation des professions libérales et plus spécifiquement du notariat au Bas-Canada) est exposée à la fin de sa lettre ouverte:

La décadence de cette profession vient naturellement de deux causes; la première, le nombre immense des notaires; la seconde cause, l'incapacité et la conduite irrégulière de plusieurs qui exercent cette profession; et c'est cette dernière cause qui porte les plus terribles coups à la profession de notaire, quoique le nombre de ces personnes soit peu considérable: car la grande majorité des notaires, est composée d'hommes recommandables par leur science et leur loyale intégrité et par une probité à toute épreuve.

Le signataire de cette première lettre met alors ses confrères, et la population en général, en garde contre les dangers de la multiplication des notaires et des médecins. Chose plus importante encore, il dénonce certains d'entre eux qui, non seulement «encombrent» leur profession, mais surtout la déshonorent. Cette thèse est en effet importante puisque c'est sur ce point précis que réagissent alors les lecteurs de La Minerve. Un débat s'ensuit qui occupera régulièrement les pages de ce journal au cours de l'année 1828 . Plusieurs lettres affluent au journal pour témoigner d'une situation que leurs auteurs jugent de plus en plus intolérable ${ }^{28}$.

On peut percevoir dans les accusations portées contre certains membres des professions libérales, jugés tantôt «ignorants», tantôt «sans mœurs», toujours sans éducation, qui déshonorent leur profession et dévalorisent par le fait même la valeur sociale d'un titre, l'une des stratégies par laquelle un groupe se forge une identité sociale. Pour les membres des professions libérales, en ce début de siècle, l'enjeu est de taille puisqu'il s'agit aussi de définir les membres de ces professions, non pas tant comme des experts ou des professionnels, mais bien comme une classe de gens supérieurs, une élite sociale légitimée à occuper une place importante dans l'espace social. Dans ce sens, le travail de construction et de constitution de ces groupes chevauche celui de la construction d'une classe sociale. L'avccat, le médecin ou le notaire se doit d'être un honnête homme pour que l'ajustement

27. La Minerve, 12 mai 1828 , p. 2-3.

28. La Minerve, 15 mai, p. 3, 19 mai, p. 2, 30 octobre, p. 2, 6 novembre, p. 4, 15 décembre 1828 , p. 3 . 
soit parfait entre le titre professionnel et la position sociale. En parlant de la profession de notaire, un lecteur de La Minerve fait d'ailleurs observer que:

Cette vaste profession qui ne devrait être exercée que par des hommes de la plus grande érudition et de mœurs irréprochables, ne voit presque rien aujourd'hui dans son sein que des hommes sans lettres et sans principes. Un vrai gentilhomme peutil embrasser cet état lorsqu'il se voit environné d'êtres d'une nature si méprisable et par leur conduite et par leur ignorance ${ }^{29}$ ?

Il va sans dire que si les porteurs de ce discours sur l'encombrement des professions libérales insistent sur le fait que ces professions accueillent des ignorants aux mœurs douteuses, c'est en fait pour réclamer de la législature qu'elle donne les moyens aux membres de ces professions d'assurer, eux-mêmes, la respectabilité de leur profession et ce, dans l'intérêt du public. On ne s'étonnera pas si les porte-parole de ce discours sont unanimes pour réclamer l'incorporation des professions libérales. Le travail d'épuration et d'exclusion entrepris par les membres de ces professions, indissociable de la trajectoire ascendante de ce groupe social, passe donc naturellement par la quête collective des pouvoirs législatifs régissant ces professions.

Dans un article de fond sur le sort des professions libérales, M. Morin, rédacteur de La Minerve, révèle les enjeux réels qui sous-tendent ce premier débat sur l'encombrement des professions libérales. Après avoir fait part des interventions qui font état de la situation déplorable où elles se trouvent, il propose ces solutions:

La législature devrait certainement intervenir pour parer aux abus, $[\ldots]$ assurer la subsistance et la respectabilité convenables de ceux qui embrassent ces états, en prévenant pour l'avenir l'introduction de personnes que leur savoir ou leurs mours ne qualifient pas suffisamment. La loi doit intervenir dans un pareil cas, les professions d'avocat, de notaire et de médecin, ne doivent pas être un champ libre, une exploitation ordinaire laissée à la compétition et ouverte pour le plus habile et le plus heureux. [...] Les avocats, les notaires et les médecins n'ont pas pour unique devoir de gagner de l'argent; ils devraient toujours avoir sous les yeux qu'ils sont des officiers publics dont la position sociale est très délicate, et la responsabilité d'autant plus grande, que ceux pour qui ils travaillent n'ont généralement aucun moyen de juger du mérite de leurs propres juges, et que leur ministère est un ministère de confiance. [...] Je voudrais donc que la législature intervienne pour assurer la respectabilité des professions libérales ${ }^{30}$.

L'auteur termine son article par quelques avis qui sont aussi révélateurs des rapports entre la position sociale et l'occupation professionnelle ${ }^{31}$.

Enfin, inutile de préciser que le collège classique, sans être mentionné dans ce discours sur l'encombrement des professions libérales, n'en demeure pas moins omniprésent, puisqu'il est le principe de la distinction entre les encombrés et les encombrants, entre l'honnête homme et l'homme ordinaire. Le rôle central de cette institution sera d'ailleurs révélé lorsqu'il s'agira de légiférer sur les demandes que renferme ce discours.

29. La Minerve, 6 novembre 1828, p. 4.

30. La Minerve, 15 décembre 1828, p. 3.

31. L'auteur associe des caractères ou dispositions personnelles aux différentes carrières. Pour les professions libérales, il faut, outre de bonnes études, des talents, de la facilité et du génie. Pour le grand. commerce, en plus de l'argent, il est nécessaire de posséder un esprit méthodique et spéculatif. Les métiers d'artisans, de laboureur ou d'ouvrier sont pour ceux qui ont des connaissances imparfaites, des bras et du courage. On voit, ici, comment s'instaurent les classements sociaux qui perdurent et apparaissent, à force de les proclamer sur toutes les tribunes, comme des cela-va-de-soi. L'auteur termine d'ailleurs son exposé par un de ces lieux communs: «Par là, chacun à sa place, et à la fin tout le monde s'en trouve mieux». 


\section{LA QUÊTE DE STATUTS SOCIAL ET PROFESSIONNEL}

Le débat sur l'encombrement des professions libérales, qui apparait à la fin des années 1820 , ne nous renseigne pas tant sur la situation sociale ou économique des avocats, des médecins ou des notaires, que sur les aspirations réelles d'un groupe en pleine ascension sociale. On ne s'étonnera pas de voir ce débat quitter les pages de La Minerve pour se transporter dans les officines du pouvoir, et plus particulièrement à la Chambre d'Assemblée où bon nombre de députés, on le sait, font eux-mêmes partie des professions libérales.

Les efforts des membres des professions libérales pour s'assurer de pouvoirs quasi absolus sur les mécanismes qui régissent leur profession, produisent les premiers résultats avec les médecins et chirurgiens. Après plusieurs échecs, c'est finalement en 1831 que sera adopté un projet de loi intitulé «Acte qui règle la profession de la médecine, de la chirurgie». Au Bas-Canada, c'est la première loi qui accorde à un corps professionnel, un statut de profession autonome. Cette loi concède aux médecins le contrôle de l'accessibilité à la profession. Elle stipule que toute personne qui désire étudier la médecine, afin d'obtenir une licence, doit d'abord subir un examen sur la langue maternelle, sur la langue latine, et montrer qu'elle est, sous tous rapports, qualifiée à entrer dans l'étude de la profession $^{32}$. En fait, le bureau médical de Montréal, créé par la loi, adopte, en 1832, des clauses qui obligent pratiquement les candidats à la profession à suivre un cours classique. Ainsi, la clause III exige «que chaque candidat à l'étude de la médecine sera obligé de répondre au bureau médical à des questions relatives à la langue maternelle, la rhétorique, la logique et la philosophie naturelle» ${ }^{33}$.

Cette loi, comme bien d'autres à cette époque, n'est pas immuable. Elle est effective jusqu'au premier mai 1837. Au moment de son expiration, le Bas-Canada traverse une crise politique grave; elle ne sera donc pas renouvelée. Ce n'est que sous le régime de l'Union, en 1847, que le parlement adoptera une loi complète et permanente.

Pourtant, cette loi temporaire demeure très importante puisqu'elle inspire les membres des professions d'avocat, de notaire et d'arpenteur dans leur démarche pour se doter des mêmes pouvoirs législatifs. Ainsi, en 1831, Dominique Mondelet, notaire et député de Montréal, présente un projet de loi concernant l'organisation du notariat. Mondelet justifie alors la nécessité d'une loi régissant le notariat, en dénonçant «l'état honteux dans lequel croupit le notariat» ${ }^{34}$, et ce, principalement à cause de l'ignorance de ceux qui exercent cette profession. Il propose alors la création d'une chambre de notaires semblable à celles que la loi a établies en France, où la capacité, l'honnêteté, enfin la conduite entière des notaires sont soumises à un examen et à une discipline rigoureuse. Le projet propose également la nomination de notaires visiteurs, afin d'établir un contrôle parfait et continu des examinateurs des candidats à la profession. Le projet mentionne que ces examinateurs ne s'informeront pas seulement des qualifications morales des aspirants, mais qu'ils veilleront à ce que ceux-ci aient les connaissances préparatoires requises pour exercer leurs fonctions. Mondelet énumère alors les connaissances nécessaires à la pratique du notariat; il s'agit de la grammaire, de la ponctuation, de la géométrie et de la connaissance des lois ${ }^{35}$.

Les débats autour du projet de loi concernant l'organisation du notariat se prolongent jusqu'en 1832, année où il est référé à un comité spécial. Un membre de ce comité, JeanJoseph Girouard, prend alors la relève de Dominique Mondelet comme défenseur de la

32. Statuts du Bas-Canada, Guillaume IV, chap. 27, 1831. I, Voir J. BERNIER, «Vers un nouvel ordre nédical: les origines de la corporation des médecins et chirurgiens du Québec», in Recherches sociographiques, vol. XXII, $\mathrm{n}^{\circ} 3,1981$, p. 316.

33. J. BERNIER, «Vers un nouvel ordre...» op. cit., p. 316.

34. Le Canadien, 7 décembre 1831, p. 1, La Minerve, 12 décembre 1831, p. 1.

35. Ibid. 
cause des notaires, après que ce dernier eut été nommé conseiller exécutif. En 1834, Girouard propose un nouveau projet de loi. Ce projet de loi se voit encore soumis à un comité spécial. Finalement, en 1836, la proposition du projet de loi de Girouard galvanise l'enthousiasme des membres de cette profession, enthousiasme dont on retrouve les traces dans les journaux de l'époque ${ }^{36}$. Cependant, le projet de loi proposé par Girouard est rejeté en 1836 pour des raisons à la fois politiques et professionnelles. Il n'en demeure pas moins que la loi régissant la profession de notaire qui est finalement adoptée par la Chambre en 1836, concerne directement l'accessibilité à la profession. Il s'agit, en fait, d'une partie du projet de loi de Girouard qui est voté sous le titre d' «Acte pour rappeler certaines parties d'une ordonnance y mentionnée qui concerne les personnes qui désirent être admises à pratiquer la loi où à pratiquer comme notaire en cette province» ${ }^{37}$. Cette loi stipule principalement que les aspirants qui ont suivi un cours d'études classiques voient leur période de cléricature écourtée.

Pour les avocats, l'année 1831 marque aussi le début d'actions visant à faire adopter une loi régissant l'organisation du barreau à la Chambre d'Assemblée. Quelques jours à peine après que le député et notaire Mondelet eut proposé son projet de loi sur l'organisation du notariat, M. Lafontaine, député et avocat, en soumet un sur l'organisation du barreau. L'élément principal de ce projet de loi réside dans l'établissement, dans les villes de Québec, Montréal et Trois-Rivières, d'un conseil choisi parmi les avocats résidents, dont les attributions garantissent l'autonomie de la profession. Une clause mentionne que «personne ne sera admis à l'étude de la loi sans faire preuve d'une éducation libérale, et de bonne mœurs, le tout à la satisfaction du conseil, qui en donnera son certificat et fera inscrire son nom sur le tableau des étudiants en droit» ${ }^{38}$. Cette clause du projet de loi entraîne d'ailleurs un débat houleux chez certains députés. Le solliciteur général $M$. Ogden, MM. Neilson et Cuvillier s'opposent à ce projet de loi, tandis que MM. Lafontaine, Duval et Dumoulin le défendent. Les détracteurs de ce projet de loi allant même jusqu'à dire qu'il rendrait légale la tyrannie d'un conseil d'avocats sur les membres de leur profession $^{39}$.

Les reproches adressés au projet de loi de Lafontaine par certains députés de la Chambre, nous indiquent que des élus sont réticents à accorder à un groupe d'individus des pouvoirs juridiques sur toute une profession. D'autre part, ils nous informent sur l'importance qu'accordent les promoteurs de ces projets de loi à la valeur de l'éducation des aspirants aux professions libérales. Il semble donc qu'il soit absolument nécessaire, pour les porte-parole de ces professions, que les futurs avocats ou notaires possèdent des qualités spécifiques qui les distinguent. Il ne s'agit pas, ici, de qualités se rapportant à une compétence technique, mais bien de dispositions culturelles. En fait, ce sont les caractéristiques constitutives de l'honnête homme que l'on souhaite le plus «incorporer» dans chaque aspirant. L'incorporation que les porte-parole des professions libérales réclament avec empressement au cours des années 1830 est avant tout celle d'un capital culturel qui confère aux membres de ces professions, et par là à la profession elle-même, des lettres de noblesse.

L'acte qui règle la profession des médecins et chirurgiens et les projets de loi proposés par Mondelet, Girouard et Lafontaine ont un but en commun; exclure des rangs de ces professions, non pas tant les charlatans, fraudeurs ou incompétents, mais surtout

36. Les notaires du district de Québec signent une lettre ouverte pour appuyer le projet de loi. Le Canadien, 22 janvier 1836, p. 3. D'autres témoignages d'appui se retrouvent dans les journaux, Le Canadien, 29 janvier 1836, p. 3, 5 février 1836, p. 3, Gazette de Québec, 30 janvier 1836, p. 3.

37. Acte 6 Guillaume IVc. $10,1836$.

38. La Minerve, 9 janvier, p. 1, 12 janvier 1832, p. 2, clause XII.

39. Gazette de Québec, 11 janvier, p. 2, 13 janvier 1832, p. 2, La Minerve, 16 janvier 1832, p. 1-2, Le Canadien, 14 janvier 1832, p. 2. 
tous ceux qui n'auraient pas la culture de l'homme accompli, en ce XIX ${ }^{\mathrm{e}}$ siècle $^{40}$. Il est ainsi beaucoup plus important (si l'on en croit les résolutions de ces projets de loi) d'exiger des candidats à la profession un certificat de bonne mœurs et une preuve qu'ils ont reçu l'éducation libérale que procure le collège classique, que de créer une école de droit encore inexistante à cette époque, ou une école de médecine pour la population canadiennefrançaise.

Le débat sur l'encombrement des professions libérales qui trouve son aboutissement logique dans les projets de loi proposés à la Chambre d'Assemblée, nous confirme clairement que l'un de ses objectifs principaux demeure toujours la valorisation de leur titre à la fois sur le marché du travail et dans l'espace social. En incitant les aspirants aux professions libérales à fréquenter un collège, on contribue à élargir la distance sociale entre les détenteurs de titres professionnels et les classes sociales dépourvues de capital culturel.

En 1847, les médecins et chirurgiens obtiennent, grâce à un acte de loi régissant leur profession, «que personne ne serait admis à l'étude de la médecine, de la chirurgie et de l'art de l'obstétrique avant d'avoir obtenu un certificat du Bureau provincial de la médecine» ${ }^{41}$. Les exigences requises sont stipulées dans cette loi. Il s'agit d'un certificat de bonne mœurs, d'une connaissance suffisante du latin, de l'histoire, de la géographie, des mathématiques, de la philosophie de la nature et d'une bonne connaissance des langues anglaise et française. On voit difficilement comment un candidat à cette profession pourrait satisfaire ces exigences sans passer par le collège classique. La même année, c'est la loi créant les chambres de notaires qui est sanctionnée. Encore là, il est stipulé que le collège classique est pratiquement obligatoire pour l'admission à la cléricature ${ }^{42}$.

Finalement, en 1849, la constitution d'un barreau indépendant couronne les efforts des avocats qui, à leur tour, pourront épurer leur profession de certains «encombrants». Avocats, médecins et notaires continuent maintenant avec des moyens que la législature leur a fournis, à raffiner ce long travail d'exclusion et d'inclusion dont ils sont, à toutes fins pratiques, le produit social.

En 1852, le président de la Chambre des notaires de Québec, M. Glackemeyer, peut alors préciser les dernières étapes de ce travail entrepris au début du siècle:

La nécessité d'avoir une éducation classique pour être admis à l'étude de la profession, une cléricature sérieuse, des études suivies et une conduite irréprochable, joints à un examen sévère achèveront de placer la profession à ce haut degré qu'elle doit occuper dans l'échelle sociale ${ }^{43}$.

\section{SYSTÈME D'ENSEIGNEMENT ET REPRODUCTION SOCIALE}

La deuxième moitié du XIX ${ }^{\mathrm{c}}$ siècle demeure une période de profonds changements pour le Québec. L'industrialisation est le phénomène le plus marquant de cette moitié de

40. Par exemple, en ce qui concerne les médecins et chirurgiens, on prend l'habitude, dès 1831 , de recevoir sans examen ceux qui ont reçu une éducation classique, et ce, bien que cette mesure ne soit pas stipulée dans la loi. Un exemple des lettres de recommandation, adressées par les médecins aux examinateurs, nous révèle d'ailleurs que, ce que l'on exige des aspirants à la médecine, ce sont avant tout des qualités morales et un comportement qui fassent honneur à la profession: «Je ne crois pas devoir le laisser partir sans le recommander particulièrement. $M$. Grammont est, comme il vous sera facile de le remarquer, très grave (il passe de beaucoup son maître en cela), c'est un grand point de gagné, car ce qui donne de la supériorité à nos médecins anglais, c'est cette figure pâle et allongée, ce phlegme que nous francs canadiens ne possédont pas». Lettre du $\mathrm{D}^{\mathrm{r}}$ Drolet au $\mathrm{D}^{\mathrm{r}}$ Painchaud citée par J. BERNIER, «Vers un nouvel...», op. cit., p. 317.

41. Statuts du Bas-Canada, Victoria, chap. 26, 1847.

42. Statuts du Bas-Canada, Victoria, chap. 21, 1847. La clause XVII stipule que l'aspirant doit passer un examen public sur sa capacité et ses qualifications et qu'il doit prouver qu'il a suivi un cours régulier d'études dans un ou plusieurs séminaires ou collèges classiques.

43. Cité par J. E. ROY, op. cit., vol. III, p. 415. 
siècle. L'idéologie montante est alors celle du libéralisme économique ${ }^{44}$. On sait maintenant que, dans la deuxième moitié du XIX ${ }^{\mathrm{e}}$ siècle, plusieurs groupes sociaux canadiens-français véhiculent un discours libéral ${ }^{45}$. En effet, le libéralisme n'est pas seulement prôné par un groupe d'hommes d'affaires; plusieurs membres des professions libérales, des journalistes et des éducateurs laïcs l'expriment avec autant d'ardeur ${ }^{46}$. D'un autre côté, à la faveur d'une grave crise politique, le clergé joue, à partir des années 1840 , un rôle primordial dans le champ de l'éducation.

On connaît la lutte que mèneront certaines élites laïques contre le monopole qu'exerce le clergé sur l'enseignement supérieur au Canada français ${ }^{47}$. Il faut dire que les intérêts des membres des professions libérales qui, comme on l'a vu, réussissent à apparaître désormais comme un groupe relativement homogène, entrent en opposition sur bien des points avec ceux du clergé. C'est sûrement dans le domaine de l'éducation que cette opposition se révèle le plus crûment. En effet, pour conserver la valeur de leurs titres scolaire et professionnel, les porte-parole des membres des professions libérales, se doivent de contrôler le système scolaire auquel ils doivent leur identité. Bien que pour ces groupes sociaux, le collège classique reste l'instrument par excellence de légitimation et de reproduction sociale, il n'en demeure pas moins que cette unique filière dans l'enseignement secondaire menace, à plus ou moins long terme, la valeur de leur titre professionnel, dans la mesure où elle contribue à augmenter le volume de ces titres dont lạ valeur repose, entre autres, sur leur raretét ${ }^{48}$.

Le discours sur l'encombrement des professions libérales qui, à la fin des années 1820 , est mis en place pour attirer l'attention sur la nécessité de créer des corporations professionnelles autonomes, afin de désencombrer ces professions de certains «encombrants», prend, à partir de la deuxième moitié du $\mathrm{XIX}^{\mathrm{e}}$ siècle, un tout autre sens. Le discours sur l'encombrement des professions libérales servira désormais d'arme contre le monopole des collèges classiques dans le système d'enseignement secondaire franco-catholique. Le discours, qui réclame la création de nouvelles filières de l'enseignement secondaire, apparaît dès lors comme une réaction de défense de certains usagers du système d'enseignement secondaire envers l'utilisation toujours plus grande du collège classique par les fils de paysans, de petits commerçants ou d'ouvriers. Si le collège classique a servi d'instance de production de groupes sociaux dans la première moitié du XIX ${ }^{\mathrm{e}}$ siècle, il est vite devenu le lieu de reproduction privilégié de ces groupes ${ }^{49}$. Toutefois, à mesure que se multiplient les collèges, un plus grand nombre de fils de paysans, de commerçants et même d'ouvriers fréquentent ces institutions. Ceux-ci, et plus particulièrement les fils d'agriculteurs, contribuent à reproduire le groupe social qu'est le clergé. Certains d'entre eux y trouvent pourtant une voie d'ascension sociale grâce à laquelle ils gagnent les rangs des professions libérales, intensifiant par le fait même l'inflation des titres sur le marché du travail ${ }^{50}$. Il s'ensuit une crainte chez les anciens détenteurs de ces titres d'une éventuelle baisse de leurs valeurs à la fois sociale et économique.

44. P.-A. LINTEAU, R. DUROCHER, J.-C. ROBERT (1979), Histoire du Québec contemporain, vol. I, Montréal, Boréal Express, p. 308.

45. F. ROY, Progrès, harmonie, liberté, Montréal, Boréal, 1988.

46. F. ROY, op. cit., p. 277.

47. R. GAGNON, «Les discours sur l'enseignement pratique au Canada français 1850-1900», in M. FOURNIER, Y. GINGRAS, O. KEEL, Sciences et médecine au Québec, Québec, IQRC, 1987, pp. 19-39.

48. L'augmentation du nombre de collèges classiques, que ne peuvent contrôler les élites laïques au $\mathrm{XIX}^{\mathrm{e}}$ siècle, n'est pas étrangère à l'augmentation des membres des professions libérales. En 1800, il existe deux collèges classiques. En 1875 , leur nombre est de vingt-quatre.

49. Claude Galarneau a réalisé une étude par échantillonnage, de 1831 à 1960 , sur les finissants du Séminaire de Québec, qui montre que les fils des membres des professions libérales en deviennent membres à leur.tour, voir C. GALARNEAU, op. cit., p. 146.

50. Un article récent sur les notables trifluviens à la fin du XIX $\mathrm{X}^{\mathrm{c}}$ siècle, nous révèle des statistiques fort intéressantes sur l'origine sociale des membres des professions libérales faisant partie de ce groupe de 
Dès les années 1850 , il devient donc urgent, selon plusieurs parlementaires et éditorialistes, de transformer le système scolaire afin de juguler le flot grandissant de nouveaux membres des professions libérales qui envahissent le marché du travail. Cette réforrne de l'enseignement passe par la mise en place d'une éducation pratique, basée sur des connaissances scientifiques et techniques, à côté d'une éducation classique. Bien que les promoteurs du discours réclamant la mise sur pied d'une autre filière dans le systèrne d'enseignement secondaire légitiment leurs revendications en invoquant le caractère hautement utilitaire et nécessaire de l'enseignement pratique dans un pays en plein développement économique, il n'en reste pas moins que l'éducation pratique instaure aussi une hiérarchie scolaire qui contribue à renforcer le statut social des professions libérales. Ainsi, les plus ardents défenseurs de l'éducation pratique reconnaissent, comme allant de soil, le statut inférieur de ce type d'enseignement. On a beau affirmer «qu'on ne s'est pas assez occupé d'écoles pratiques ${ }^{51}$, et proclamer «qu'il faudrait une éducation plus pratique pour nous rendre capables de tirer parti des avantages et des richesses de notre pays $^{52}$, ou encore constater «qu'il est absolument nécessaire de créer des écoles pratiques pour former des ingénieurs, des mécaniciens, des géomètres ${ }^{53}$, malgré toutes ces déclarations d'intention, l'enseignement pratique reste un enseignement dominé, pensé en fonction d'accueillir les enfants des classes dominées de la société.

L'avocat P.J.O. Chauveau, surintendant de l'Instruction publique de 1855 à 1866, puis premier ministre du Québec et ministre de l'Instruction publique de 1867 à 1872 , grand défenseur de l'enseignement pratique, décrit bien, dans son rapport de 1872, à titre de ministre de l'Instruction publique, les fonctions sociales de l'éducation pratique:

Tout le monde aujourd'hui apprécie l'importance de ces établissements [académies commerciales et écoles techniques]. C'est là que l'enfant reçoit une éducation pratique, appropriée à toutes les classes de la société, principalement aux classes ouvrières et mercantiles. C'est là que se forment tous les membres de cette classe moyenne qui est en quelque sorte l'âme vive de la nation, qui en fait la prospérité lorsqu'elle se montre à la hauteur de sa mission ${ }^{54}$.

Si les académies forment la classe moyenne de la société, on devine aisément quels établissements produisent la classe supérieure. Chauveau passe d'ailleurs à la description d'une quatrième classe d'institutions, celle des collèges. Ne contestant nullement la supériorité de ce type d'enseignement, Chauveau suggère pourtant que «la province compte un assez grand nombre de ces institutions, trop peut-être pour le chiffre de notre population, et si l'on ne perd pas de vue le besoin vital d'une éducation surtout pratique dans un jeune pays comme le nôtre» ${ }^{55}$.

dirigeants locaux. Sur les 60 notables membres des professions libérales, l'auteur a repéré l'origine sociale de 49 d'entre eux. Dix-sept ont un père agriculteur, onze sont des fils de membres des professions libérales et onzt également fils de marchands. C'est lorsque l'on regarde le statut socio-professionnel des pères des 173 notables répertoriés par l'auteur de cet article que l'on est saisi par la forte tendance des fils des professions libérales à exercer la même profession que leur père. Sur les quinze fils de membres des professions libérales, 11 exercent ce type de profession. Ces statistiques tendent à confirmer notre thèse. Elles indiquent, en effet, que les membres des professions libérales envoient leurs enfants au collège pour leur assurer une position sociale équivalente à celle qu'ils occupent. Elles nous montrent également que les collèges demeurent toujours une filière de promotion sociale importante pour les fils d'agriculteurs, ce qui ne peut manquer d'alarmer les membres des professions libérales. Voir F. GUÉRARD, «Les notables trifluviens au dernier tiers du XIX ${ }^{\mathrm{e}}$ siècle: stratégies matrimoniales et pratiques distinctives dans un contexte d'urbanisation» in RHAF, vol. XLII, $\mathrm{n}^{\circ} 1$, été 1988 , pp. 27-46.

51. G. OUIMET, Rapport du ministre de l'Instruction publique de la province de Québec pour l'année 1872 et une partie de 1873, Québec, p. ix.

52. L. O. DAVID, in L'Opinion publique, 5 février 1870, p. 2.

53. Débats de l'Assemblée législative du Québec (DALQ), H. G. JOLY, 16 janvier 1874.

54. P. J. O. CHAUVEAU, Rapport du ministre de l'Instruction publique de la province de Québec pour l'année 1871-1872, Québec, p. viii. (C'est nous qui soulignons).

55. Ibid. 
Son successeur, aux postes de premier ministre et de ministre de l'Instruction publique, l'avocat Gédéon Ouimet, explique, quant à lui, dans le rapport du ministre de l'Instruction publique de 1873, les conséquences de l'encombrement des professions libérales, et ce, pour mieux insister sur la nécessité d'une éducation pratique:

C'est rendre un mauvais service à la société dans un jeune pays comme le nôtre, que de faciliter l'accès des maisons d'éducation supérieure. On délaisse ainsi un grand nombre de jeune gens qui, après un cours d'études classiques, se trouvent impropres à toutes espèces de carrière, si leurs aptitudes ou les circonstances ne leur permettent pas d'étudier une profession libérale; et ceux qui ont passé par là savent seuls tous les mécomptes, toutes les tribulations qui attendent à son entrée dans la vie réelle le jeune homme ainsi déclassés6.

Ainsi, il est clair que pour les ministres de l'Instruction publique, l'éducation pratique est perçue comme un moyen de restreindre l'accès à l'éducation supérieure. Pour les porte-parole des médecins, avocats ou notaires, c'est en invoquant l'encombrement des professions libérales qu'ils dénonceront les dangers d'une éducation trop prodiguée. En 1899 , dans un discours intitulé «Les dangers de l'instruction classique à outrance» ${ }^{57}$, un porte-parole important des notaires, le président de la Chambre des notaires, Léandre Bélanger, constate que l'instruction supérieure qu'offrent les collèges a généralement pour but de former des prêtres, des médecins, des avocats, des notaires. Selon lui, «comme il ne faut dans la société qu'un nombre restreint et limité d'hommes de profession, et comme d'autre part il y a un courant tendant à nous en préparer un nombre illimité» ${ }^{58}$, l'encombrement des professions libérales est inévitable. C'est toutefois lorsqu'il déplore l'utilisation par les classes moyennes de l'enseignement supérieur que le président de la Chambre des notaires révèle l'une des fonctions importantes du discours sur l'encombrement des professions libérales. En effet, celui-ci affirme sans ambages que:

Les hommes de profession, ici, particulièrement sont les fils de parents qui n'ont pu, tout en s'imposant les plus grands sacrifices, que faire faire à leurs enfants les études nécessaires pour les faire admettre à ces professions et qui n'ont plus ensuite les moyens de les soutenir jusqu'à ce qu'ils soient arrivés à se faire une certaine clientèle qui leur permettra de vivre sur un ton convenable à leur état. Que pensezvous que ces hommes fassent, je vous le demande? [...] Voilà ce à quoi ne songent pas assez les parents de la classe moyenne, qui trop souvent s'épuisent en sacrifices inutiles pour leurs fils, dont ils réussissent [...] qu'à rendre le sort constamment précaire, la moralité fréquemment équivoque, et la vie malheureusement désordonnée. Enfin, les parents font de la besogne imprudente, qui dédaignent pour leurs enfants, la condition où ils mènent eux-mêmes une vie honorable et paisible, les nourissent dans l'idée de sortir de la sphère paternelle [...] Cette quantité de jeunes gens que les sacrifices de leurs familles ont doté d'une instruction complète se précipitent dans ce champ étroit qu'ils encombrent, pressés les uns contre les autres, se disputant à outrance les clients et les places, ils n'arrivent qu'à déprimer la rémunération moyenne de leur classe.

56. G. OUIMET, Rapport du ministre de l'Instruction publique de la province de Québec pour l'année 1872-1873, Québec, p. viii. On pourrait continuer la citation des discours qui reproduisent ces mêmes énoncés. Il ne s'agit pas, ici, d'un discours isolé. À cette époque, c'est devenu presqu'un lieu commun que d'affirmer ces thèses sur l'éducation classique et pratique. En 1871, le discours de l'avocat libéral François Langelier, devant les électeurs du comté de Bagot, reprend les mêmes 'thèmes: «L'éducation supérieure est une excellente chose, mais il ne faut pas qu'elle soit trop prodiguée. Car d'abord elle cessera d'être supérieure. Elle produira des demi-savants, des individus déclassés, qui viendront grossir le nombre, déjà trop grand, de ceux qui végètent dans les professions libérales au lieu de faire bénéficier de leur travail et de leurs talents l'agriculture, le commerce et l'industrie». Cité dans Le Pays, 9 juin 1871, p. 2.

57. L. BÉLANGER, «Les dangers de I'instruction classique à outrance» in Revue du notariat, vol. II, 1899-1900, pp. 235-239.

58. L. BÉLANGER, op. cit., p. 236. 
Le privilège de l'instruction a presque disparu. La capacité moyenne utile, estimable, qui était bien appointée jadis, parce qu'elle constituait une rareté, se paie aujourd'hui à un prix dérisoire ${ }^{59}$.

Quelques années auparavant, un éditorial, dans la revue médicale la plus importante au Québec, L'Union médicale du Canada, intitulée «Trop de médecins», se prononce sur les causes de l'encombrement des professions libérales. L'éditorialiste écrit:

La multiplication excessive de nos collèges classiques a mis l'éducation secondaire à la portée de tous et la conséquence en est qu'un grand nombre des élèves qui fréquentent ces collèges ne présentent guère les aptitudes nécessaires à une éducation supérieure. Fils de cultivateurs, ils rougiraient d'aller tenir le manche de la charrue après avoir pâli sur les livres. Aptes ou non, ils entendent bien devenir notaires, avocats ou médecins. Si les directeurs de nos collèges prenaient la chose en considération, et cherchaient à éloigner des études professionnelles les sujets qui n'ont pas les aptitudes nécessaires, ils nous rendraient un fier service. Que de jeunes gens auraient fait d'excellents agronomes ou de bons industriels qui ne sont aujourd'hui que des demi-médecins! Avec leur sens pratique avant tout, nos compatriotes d'origine anglaise calculent mieux que nous les chances de l'avenir. Aussi savent-ils diriger leurs enfants vers le génie civil et la haute industrie $[\ldots]^{60}$.

L'industrialisation qui gagne le Québec durant cette période conditionne bien entendu plusieurs groupes sociaux, au Canada français, à promouvoir l'enseignement pratique. II ne faut pas oublier pourtant que pour les membres des professions libérales, il s'agit aussi de renforcer leur position dans la structure sociale que garantissent de moins en moins leurs titres scolaire et professionnel. Si l'argument majeur pour tenter d'imposer une transformation du système d'enseignement est celui qui lie, dans un rapport de cause à effet, le système d'éducation et le développement économique, l'encombrement des professions libérales et le désencombrement qu'offrirait la mise en place d'une éducation pratique au côté d'une éducation classique sont presque toujours invoqués dans ce discours. Ainsi en 1874, un autre avocat A. G. Joly, député de Lotbinière, lance en Chambre:

Il est pénible de voir que dans notre province, l'éducation pratique est entièrement sacrifiée à l'éducation classique; nous avons un trop grand nombre de collèges où la jeunesse vient se bourrer de grec et de latin, ce qui fait que toutes les professions libérales sont encombrées ${ }^{61}$.

Quelques années auparavant, en 1868, le journal libéral Le Pays dresse un bilan du système d'éducation destiné aux Canadiens français. Il s'agit, ici, d'un exemple typique des arguments par lesquels on s'attaque au monopole des collèges dans l'enseignement secondaire:

On projette en ce moment de faire un nouveau collège classique. Nous nous élevons de toute notre force contre ce moyen extra-surabondant de produire des inutilités. Le pays est inondé de collèges classiques, et non seulement le besoin ne se fait pas sentir d'en avoir d'autres, mais encore de convertir bon nombre de ceux qui existent en académies appropriées à notre position économique, à nos ressources, à notre avenir [...] C'est encore la jeunesse des collèges qui n'a pas d'avenir avec son bagage de latin mal appris, de français plus mal appris encore, de son ignorance de l'anglais et de toutes les branches d'instruction qui seules peuvent offrir des carrières avantageuses. Ce qu'il nous faut ce sont des ingénieurs, des géologues [...] qu'il y ait des collèges classiques, c'est très bien, mais qu'ils n'existent que pour faire équilibre et non comme monopole [...] Nous voulons qu'il y ait à côté

59. L. BÉLANGER, op. cit., p. 237-238.

60. L'Union médicale du Canada, vol. XIV, mars 1885, p. 142.

61. $D A L Q, \mathrm{H}$. G. JOLY, 16 janvier 1874. 
d'elle [éducation classique」des écoles pour les sciences et les arts qui absorbent aujourd'hui presque toute la vigueur intellectuelle des peuples civilisés ${ }^{62}$.

Il est à noter que si le collège classique apparaît, dans ce discours, comme l'une des causes de l'encombrement des professions libérales, c'est avant tout par le fait qu'il exerce un monopole sur l'enseignement secondaire, comme le signale si bien l'éditorialiste du journal Le Pays. Il n'est donc pas paradoxal de constater que les membres des professions libérales persistent dans leur lutte pour exiger des aspirants à ces professions qu'ils passent par les collèges. Les membres des professions libérales intensifient également leur utilisation du collège dans leurs stratégies de reproduction sociale ${ }^{63}$. Finalement, il est important de mentionner que malgré ce discours qui souligne la surabondance des collèges et la nécessité vitale d'écoles de sciences appliquées, bien peu de gens enverront leurs enfants, à la fin du XIX ${ }^{\mathrm{e}}$ siècle, à l'École polytechnique de Montréal, fondée, en 1873, à la faveur de ce discours ${ }^{64}$.

Tout nous permet de supposer que le discours sur l'encombrement des professions libérales, à partir de la deuxième moitié du $\mathrm{XIX}^{\mathrm{c}}$ siècle, expose les principes de la stratégie employée par les détenteurs d'un titre scolaire et professionnel afin d'en éviter la dévaluation qu'ils jugent imminente. En dénonçant la multiplication des collèges classiques ou encore les dangers d'une éducation supérieure ouverte à tous, et en promouvant l'enseignement pratique, ceux dont la reproduction sociale était assurée principalement par le collège, réagissent à l'entrée dans la course et dans la concurrence pour les titres scolaires d'un plus grand nombre d'individus appartenant aux classes sociales démunies culturellement. La mise en place d'une nouvelle filière dans l'enseignement secondaire, comme celle de l'éducation pratique, hiérarchiquement inférieure à celle de l'éducation classique, pourra, espère-t-on, prendre en charge les fils d'agriculteurs (enseignement agricole), les fils de petits marchands et de petits commerçants (académies commerciales), les fils d'ouvriers ou d'artisans (écoles techniques).

\section{CONCLUSION}

Un discours persistant, tout au long du XIX ${ }^{\mathrm{e}}$ siècle, est certainement celui qui fait état de l'encombrement des professions libérales. Véhiculé tout d'abord par certains porteparole de ces professions, ce discours apparaît, dès son origine, pour signaler que la surabondance des membres des professions libérales affecte, bien sûr leurs revenus, mais aussi et surtout leur valeur sociale. Ce discours dit moins qu'il y a trop de médecins, d'avocats et de notaires, qu'il ne révèle que les «honnêtes» membres de ces professions sont soudainement envahis par des individus ne possédant pas les vertus de l' «honnêteté». Ce discours réclame donc les moyens juridiques de contrôler l'accessibilité aux professions libérales. Il témoigne d'un effort collectif, entrepris par plusieurs porte-parole de ces professions, pour assurer l'homogénéité des dispositions culturelles et sociales des membres

62. Le Pays, 20 juin 1868 , p. 2.

63. Voir les statistiques établies par Claude Galarneau dans Les collèges... op. cit., pp. 142-145.

64. L'École polytechnique de Montréal décernera, en moyenne, de 1877 à 1899, trois diplômes par année. De plus, entre 1855 et 1870 , plusieurs collèges classiques qui s'étaient convertis en collèges, dits industriels, ou avaient inauguré un enseignement pratique parallèle à l'éducation classique, abandonneront ce type d'enseignement ou redeviendront des collèges classiques traditionnels, entre 1870 et 1900, compte-tenu du peu de popularité des études pratiques. Voir à ce sujet L. P. AUDET, Le système scolaire au Québec, Montréal, Beauchemin, 1967, pp. 25-26. Soulignons, à l'appui de notre thèse, que les membres des professions libérales, qui forment le groupe qui utilise le plus fortement l'enseignement supérieur pour assurer leur reproduction sociale, ne dirigent pratiquement pas leurs enfants à l'École polytechnique de Montréal. Ainsi de 1873 à 1885 , l'École fait voir dans ses livres 119 inscriptions. Quatorze de ces étudiants sont des fils de médecins, d'avocats ou de notaires. De plus, seulement 49 de ces étudiants inscrits obtiendront un diplôme d'ingénieur, de ceux-ci, 6 ont un père membre des professions libérales. Archives de l'École polytechnique de Montréal. 
des professions libérales qui pourront désormais apparaître comme des personnes, dignes d'accéder aux positions dominantes dans la société canadienne-française.

$\mathrm{Si}$, au début du XIX $\mathrm{X}^{\mathrm{e}}$ siècle, le discours sur l'encombrement des professions libérales se comprend en le rapportant à cet effort collectif de revendication et d'unification auquel il doit toute sa force, à partir de la deuxième moitié du XIX ${ }^{e}$ siècle, il s'inscrit dans un débat beaucoup plus large. En effet, le discours qui fait mention de l'encombrement des professions libérales dénonce désormais le monopole des collèges classiques sur l'enseignement secondaire. Ce discours peut être saisi en le rapportant aux stratégies de reproduction sociale de groupes richement pourvus en capital culturel. En exigeant l'érection d'académies commerciales, d'écoles techniques et d'écoles de sciences appliquées aux côtés des collèges, c'est beaucoup moins une stratégie de reconversion du capital culturel en une autre espèce (économique par exemple), à laquelle les membres des professions libérales s'adonnent, qu'à une stratégie de conservation de la valeur socio-économique de leurs titres scolaire et professionnel ${ }^{65}$.

\author{
Robert Gagnon \\ Département de sociologie \\ Université de Montréal \\ C.P. 6128 , Succ. «A» \\ Montréal (Québec) \\ Canada $\mathrm{H} 3 \mathrm{C} 3 \mathrm{~J} 7$
}

\title{
RÉSUMÉ
}

Cet aiticle a pour objet le discours sur l'encombrement des professions libérales au XIX ${ }^{e}$ siècle. Au lieu de s'interroger sur le nombre des avocats, médecins ou notaires, afin de déterminer si ces professions connaissent un «encombrement» au cours du XIX ${ }^{e}$ siècle, l'auteur suggère plutôt que l'encombrement des professions libérales dont font état plusieurs membres de ces professions est en fait une construction sociale. Dans la première moitié du XIX ${ }^{\mathrm{e}}$ siècle, ce discours est relié au travail de désignation entrepris par les membres des professions libérales afin de se doter d'une identité sociale commune: celle de l'honnête homme. Plus tard, dans la deuxième moitié du XIX ${ }^{\mathrm{e}}$ siècle, ce discours remplit une tout autre fonction sociale. L'auteur rapporte le discours sur l'encombrement des professions libérales aux stratégies de reproduction de groupes désormais formés. La multiplication des collèges et le monopole de l'éducation classique sur le système d'enseignement secondaire au Québec, en prédisposant les étudiants à la prêtrise ou aux professions libérales, menacent la valeur sociale et économique de ces titres professionnels. L'encombrement des professions libérales est alors invoqué pour restreindre l'accès aux collèges classiques, notamment en promouvant la mise en place d'une nouvelle filière dans l'enseignement secondaire: celle de l'éducation pratique.

\section{SUMMARY}

The cobject of this paper is the discourse on the saturation of the liberal professions in the 19th century. Instead of locking into the number of lawyers, physicians and notaries to determine if these professions were 'saturated' during the 19th century, the author suggests rather that the saturation of the liberal professions as reported by a number of members of these professions is in fact a social construction. In the first half of the 19th century, this discourse is linked to a work of designation undertaken by members of the liberal professions in order to give themselves a common social identity: that of the gentleman (honnete homme). Later, in the second half of the 19th century, this discourse fulfilled a completely different social function. The author relates the discourse on the saturation of the liberal professions to the reproduction strategies of the groups formed at that time. The multiplication of colleges and the monopoly of classical education over the secondary education system in Quebec, thereby predisposing students to the priesthood or the liberal professions, threatened the social and economic value of these professional titles. The saturation of the liberal professions was therefore brought forward as an argument for limiting access to classical colleges, particularly by promoting the creation of a new channel in secondary education - practical education.

65. Ce qui pour certains sociologues semble être le cas pour le début du $\mathrm{XX}^{\mathrm{e}}$ siècle. Voir: $\mathrm{F}$. DESCARRIES-BÉLANGER, M. FOURNIER, L. MAHEU, «Le frère Marie-Victorin et les «petites sciences» in Recherches Sociographiques, janvier-avril 1979, vol. XX, $\mathrm{n}^{\circ} 1, \mathrm{pp} .7-39$. 


\section{RESUMEN}

Este artículo tiene por objeto el discurso sobre la saturación de las profesiones liberales en el siglo XIX. En lugar de interrogarse sobre el número de abogados, médicos o notarios, con el fin de determinar si estas profesiones conocen una «saturación» en el curso del siglo XIX, el autor sugiere más bien que la saturación de las profeciones liberales de la cual dan cuenta varios miembros de esas profesiones es en el hecho una construcción social. En la primera mitad del siglo XIX, este discurso está ligado al trabajo de designación emprendido por los miembros de las profesiones liberales con el fin de dotarse de una identidad social común: aquella del hombre honesto. Más tarde, en la segunda mitad del siglo XIX, este discurso cumple una función social muy diferente. El autor relaciona el discurso sobre la saturación de las profesiones liberales a las estrategias de reproducción de los grupos ya formados. La multiplicación de colegios y el monopolio de la educación clásica sobre el sistema de enseñanza secundaria en Quebec, predisponiendo a los estudiantes ya sea al sacerdocio o a las profesiones liberales, amenazan el valor social y económico de esos títulos profesionales. La saturación de las profesiones liberales se invoca por lo tanto para restringir el acceso a los colegios clásicos, notablemente promoviendo la puesta en marcha de una nueva filial en la enseñanza secundaria: la educación práctica. 